\title{
Critical behavior of charged dilaton black holes in AdS space
}

\author{
Amin Dehyadegari ${ }^{1}$ and Ahmad Sheykhi ${ }^{1,2,3, *}$ \\ ${ }^{1}$ Physics Department and Biruni Observatory, Shiraz University, Shiraz 71454, Iran \\ ${ }^{2}$ Research Institute for Astronomy and Astrophysics of Maragha (RIAAM), \\ P.O. Box 55134-441, Maragha, Iran \\ ${ }^{3}$ Max-Planck-Institute for Gravitational Physics (Albert-Einstein-Institute), 14476 Potsdam, Germany
}

(Received 26 February 2020; accepted 21 August 2020; published 9 September 2020)

\begin{abstract}
We revisit critical behavior and phase structure of charged anti-deSitter (AdS) dilaton black holes for arbitrary values of dilaton coupling $\alpha$, and realize several novel phase behavior of this system. We adopt the viewpoint that cosmological constant (pressure) is fixed and treat the charge of the black hole as a thermodynamical variable. We study critical behavior and phase structure by analyzing the phase diagrams in $T-S$ and $q-T$ planes. We numerically derive the critical point in terms of $\alpha$ and observe that for $\alpha=1$ and $\alpha \geq \sqrt{3}$, the system does not admit any critical point, while for $0<\alpha<1$, the critical quantities are not significantly affected by $\alpha$. We find that unstable behavior of the Helmholtz free energy for $q<q_{c}$ exhibits a first order (discontinuous) phase transition between small and large black holes for $0 \leq \alpha<1$, where $q_{c}$ is the value of charge at the critical point. For $1<\alpha<\sqrt{3}$ and $q>q_{c}$, however, a novel first order phase transition occurs between small and large black hole, which has not been observed in the previous studies on phase transition of charged AdS black holes.
\end{abstract}

DOI: $10.1103 /$ PhysRevD.102.064021

\section{INTRODUCTION}

Phase transition is certainly one of the most intriguing and interesting phenomena in the thermodynamic description of black holes which may shed light on the nature of quantum gravity. In particular, the investigations on the black hole phase structure/transition in an asymptotically AdS spacetime have received considerable attentions in the past years. This is mainly due to the remarkable duality between gravity in an $(n+1)$-dimensional AdS spacetime and the conformal field theory living on the boundary of its $n$-dimensional spacetime, (AdS/CFT) correspondence. Perhaps, one of the earliest studies in this direction was done by Hawking and Page [1], who disclosed that there is indeed a first order phase transition, latter named HawkingPage phase transition, between the thermal radiation and the stable large Schwarzschild black hole with spherical horizon in the background of AdS spacetime. Later, Witten discovered [2] that this phase transition can be interpreted in the AdS/CFT duality as the confinement/deconfinement phase transition of the strongly coupled gauge theory.

\footnotetext{
*asheykhi@shirazu.ac.ir
}

Published by the American Physical Society under the terms of the Creative Commons Attribution 4.0 International license. Further distribution of this work must maintain attribution to the author(s) and the published article's title, journal citation, and DOI. Open access publication funded by the Max Planck Society.
Recently, it has been shown that for charged AdS black hole a second and first order phase transitions occur between small and large black holes in an extended phase space which resembles the liquid-gas phase transition in the usual van der Waals liquid-gas system $[3,4]$. In an extended thermodynamic phase space, the cosmological constant (AdS length) is considered as a thermodynamic pressure which can vary, and its corresponding conjugate quantity is the thermodynamic volume of the black hole. Taking into account the variation of pressure in the first law, one observes that the mass of AdS black hole is equivalent to the enthalpy [5]. In the recent years, thermodynamic phase transitions in an extended phase space have been explored for various types of black holes in AdS space (see Refs. [6-22] and references therein). Reference [23] performed a stability analysis of charged AdS dilaton black hole by considering the second derivative of mass with respect to entropy. It was shown that the black holes are thermodynamically stable for small $\alpha$, while for large $\alpha$ black hole becomes unstable [23]. The studies on phase transition in such black hole have been carried out from both thermal and dynamical point of view [24], where the cosmological constant appears as a thermodynamical variable. They found that for small dilaton coupling, $\alpha \approx 0.01$, the system resembles the van der Waals fluid behavior, while for $\alpha>1$, the $P-v$ diagram of the system deviates and new phenomena beyond the van der Waals liquid-gas-like appears [24]. Recently, a novel phase behavior represents a small/large black hole zeroth-order 
phase transition, in an extended phase space with varying cosmological constant, has been observed for charged dilaton black holes, where the geometry of spacetime is not asymptotically AdS [25].

Another possible approach to study thermodynamic phase structure of black hole is to consider the variation of electric charge of the black hole, while the cosmological constant (AdS length) is kept fixed. With regard to this perspective, the critical behavior and phase transition of charged AdS black holes were investigated in a fixed AdS geometry, indicating that it exhibits the small/large black hole phase transition of van der Waals type [26-28]. Interestingly enough, it has been realized [26] that the phase transition of charged AdS black hole can occur in $Q^{2}-\Psi$ plane, where $\Psi=1 / 2 r_{+}$is the conjugate of $Q^{2}$, without extending the phase space. Indeed, in this alternative perspective, the relevant response function clearly signifies the stable and unstable region. Also there still exist a deep analogy between critical phenomena and critical exponents of the system with those of van der Waals liquidgas system [26]. The advantages of this new approach is that one do not need to extend the phase space by treating the cosmological constant as a thermodynamical variable which may physically make no sense [26]. It has been confirmed that this new approach also works in other gravity theories $[29,30]$ as well as in higher spacetime dimensions [31]. Recently, the universality class and critical properties of any AdS black hole, independent of spacetime metric, via an alternative phase space has been explored [32]. It was shown that the values of critical exponents for generic black hole are the same as ones of the van der Waals fluid system [32]. For Born-Infeld AdS black holed in four dimensional spacetime, we analytically calculated the critical point by studying the behavior of specific heat in a fixed AdS geometry [33]. Furthermore, the interesting reentrant phase transition of Born-Infeld AdS black hole has been investigated in the thermodynamic phase space [33]. The structure of charged black holes has been explored by employing the Ruppeiner geometry [34] in which the charge is allowed to vary.

In this paper, we study thermodynamic properties of charged AdS dilaton black hole including the phase transition and critical behavior in $(3+1)$-dimensional spacetime. Our work differs from [24] in that we keep the cosmological constant as a fixed quantity and treat the charge of the black hole as a thermodynamic variable, while the authors of [24] extended the phase space by treating cosmological constant as a variable. Following [33], we first identify different types of charged AdS dilaton black hole depending on the behavior of heat capacity at constant charge for arbitrary values of the dilaton-electromagnetic coupling constant $\alpha$ and fixed AdS length (cosmological constant). This has not been addressed in the previous studies $[23,24]$. As we shall see, the behavior of black hole temperature crucially depends on $\alpha$ for the small entropy, such that black hole has different behaviors for $0<\alpha<$ $1 / \sqrt{3}$ and $1 / \sqrt{3}<\alpha$. Besides, we calculate the critical point numerically for various values of $\alpha$ in $T$-S plane. When $\alpha=1$ and $\alpha \geq \sqrt{3}$, we cannot realize any critical point in the system which has not been reported in the previous investigations on phase transition of charged AdS black holes. Besides, for $0 \leq \alpha<1$, we observe a small/large first order phase transition for $q<q_{c}$, while similar behavior is seen for $1<\alpha<\sqrt{3}$ provided $q>q_{c}$. Indeed, there is a novel first order phase transition in the range of $1<\alpha<\sqrt{3}$ which has not previously been observed. Finally, the phase diagram for charged AdS dilaton black hole is constructed in $q-T$ plane.

This paper is structured in the following manner. In Sec. II, a brief overview on thermodynamics of the fourdimensional charged dilaton black hole in the AdS background is given. In Sec. III, we investigate critical behavior of charged dilaton AdS black holes by studying the specific heat at constant electric charge in $T$-S plane. In Sec. IV, we use the Helmholtz free energy to determine the possible phase transition in the system. Finally, we summarize the main results Sec. V.

\section{CHARGED DILATON BLACK HOLES IN AdS SPACE}

We start with a brief review on charged AdS black holes in dilaton gravity and calculate the associated conserved and thermodynamic quantities. Exact charged dilaton black hole solutions in the background of (A)dS space in four [35] and higher dimensions [36,37] have been presented by Gao and Zhang. Thermodynamics of these solutions has been investigated in $[23,38]$. The four-dimensional action of Einstein-Maxwell gravity coupled to a dilaton field is $[23,35]$

$$
\begin{aligned}
\mathcal{S}= & -\frac{1}{16 \pi} \int d^{4} x \sqrt{-g}\left(\mathcal{R}-2(\nabla \varphi)^{2}-V(\varphi)\right. \\
& \left.-e^{-2 \alpha \varphi} F_{\mu \nu} F^{\mu \nu}\right),
\end{aligned}
$$

where $\mathcal{R}$ is the Ricci scalar curvature, $\varphi$ is the dilaton field and $V(\varphi)$ is the dilaton potential. Herein, the electromagnetic field tensor $F_{\mu \nu}$ is defined in terms of the gauge field $A_{\mu}$ via $F_{\mu \nu}=\partial_{\mu} A_{\nu}-\partial_{\nu} A_{\mu}$. For an arbitrary value of the dilaton coupling strength $\alpha$ in AdS space, the dilaton potential is chosen to take the following form $[35,39]$

$$
\begin{aligned}
V(\varphi)= & \frac{2 \Lambda}{3\left(\alpha^{2}+1\right)^{2}}\left[8 \alpha^{2} e^{\left(\alpha^{2}-1\right) \varphi / \alpha}-\left(\alpha^{2}-3\right) e^{2 \alpha \varphi}\right. \\
& \left.+\alpha^{2}\left(3 \alpha^{2}-1\right) e^{-2 \varphi / \alpha}\right],
\end{aligned}
$$

where $\Lambda$ is the cosmological constant that relates to the AdS radius $l$ as $\Lambda=-3 / l^{2}$. The potential given in Eq. (2) shows that the cosmological constant $\Lambda$ is coupled to the dilaton 
field $\varphi$ in a nontrivial way. When the coupling constant $\alpha= \pm 1 / \sqrt{3}, \pm 1, \pm \sqrt{3}$, the dilaton potential in Eq. (2) is indeed the supersymmetry (SUSY) potential of string theory. Note that in the absence of the dilaton field, i.e., $V(\varphi=0)=2 \Lambda$, the action Eq. (1) reduces to the usual Einstein-Maxwell theory with cosmological constant. In $3+1$ dimensions, the line element of a static spherically symmetric spacetime is written

$$
d s^{2}=-f(\rho) d t^{2}+\frac{d \rho^{2}}{f(\rho)}+\rho^{2} R^{2}(\rho) d \Omega^{2},
$$

where $d \Omega^{2}$ is the metric of the 2-dimensional unit sphere with volume $\omega=4 \pi$ and the metric functions $f(\rho)$ and $R(\rho)$ are given by [23]

$$
\begin{gathered}
f(\rho)=\left(1-\frac{b}{\rho}\right)^{\gamma}\left[\left(1-\frac{b}{\rho}\right)^{1-2 \gamma}\left(1-\frac{c}{\rho}\right)+\frac{\rho^{2}}{l^{2}}\right], \\
R^{2}(\rho)=\left(1-\frac{b}{\rho}\right)^{\gamma},
\end{gathered}
$$

where $b$ and $c$ are integration constants and $\gamma=$ $2 \alpha^{2} /\left(\alpha^{2}+1\right)$. Also, the dilaton field and the only nonvanishing component of the gauge field $A_{\mu}$ are obtained as [23]

$$
\varphi(\rho)=\frac{\sqrt{\gamma(2-\gamma)}}{2} \ln \left(1-\frac{b}{\rho}\right), \quad A_{t}=-\frac{q}{\rho},
$$

where $q$, an integration constant, is the charge parameter which is related to $b$ and $c$ via the following relation

$$
q^{2}=\frac{b c}{\alpha^{2}+1} .
$$

For $\alpha \neq 0$, these solutions become imaginary in the range of $0<\rho<b$, so this region should be excluded from the spacetime. One may also have a close look on the expansion of $V(\varphi)$. Given $\varphi(\rho)$ at hand, it is a matter of calculation to show that for small $\alpha$,

$V(\varphi)=2 \Lambda+4 \Lambda \alpha^{2}\left\{\frac{b(\rho-7 b / 6)}{\rho^{2}(1-b / \rho)^{2}}+\ln (1-b / \rho)\right\}+\mathcal{O}\left(\alpha^{4}\right)$,

which implies that, in the presence of dilaton field, the leading correction term to the cosmological constant is of order $\alpha^{2}$. The black hole event horizon is located at $\rho=\rho_{+}$ which is determined by the largest real root of $f\left(\rho_{+}\right)=0$. The mass and electric charge of the dilaton AdS black hole per unit volume $\omega$ are [23]

$$
M=\frac{1}{8 \pi}\left(c-b \frac{\alpha^{2}-1}{\alpha^{2}+1}\right), \quad Q=\frac{q}{4 \pi},
$$

where the expression of $M$ is derived in the Appendix. Also, the other associated thermodynamic quantities, such as the Hawking temperature $T$, entropy $S$, and electric potential $U$, are

$$
\begin{gathered}
T=\frac{1}{4 \pi \rho_{+}}\left(1-\frac{b}{\rho_{+}}\right)^{1-\gamma}\left\{1+\frac{\rho_{+}}{l^{2}}\left[3 \rho_{+}+2 b(\gamma-2)\right]\right. \\
\left.\times\left(1-\frac{b}{\rho_{+}}\right)^{2(\gamma-1)}\right\}, \\
S=\frac{\rho_{+}^{2}}{4}\left(1-\frac{b}{\rho_{+}}\right)^{\gamma}, \quad U=\frac{q}{\rho_{+}},
\end{gathered}
$$

where entropy $S$ is written per unit volume $\omega$. It is easy to verify that the first law of black hole thermodynamics

$$
d M=T d S+U d Q
$$

is satisfied on the event horizon [23].

It is worthwhile to mention that in the absence of the dilaton filed $(\alpha=0)$, the solutions reduce to the well-known four-dimensional Reissner-Nordstrom (RN)-AdS black hole. It is also notable to mention that these solutions are even functions in $\alpha$. In the next section, we study the critical behavior of dilaton AdS black hole in the phase space.

\section{CRITICAL BEHAVIOR OF CHARGED DILATON AdS BLACK HOLE}

In this section we are going to investigate the effects of the dilaton field on the critical behavior of charged dilaton AdS black hole. To end this, we analyze behavior of the specific heat at constant charge

$$
C_{q}=T\left(\frac{d S}{d T}\right)_{q}
$$

where $l$ and $\alpha$ are also fixed. The sign of this quantity determines the local thermodynamic stability, i.e., the stability (instability) is accompanied by $C_{q}>0\left(C_{q}<0\right)$. To see the influence of the dilaton field $(\alpha)$ on $C_{q}$, we plot the behavior of the temperature as a function of entropy in Fig. 1 for different values of $\alpha$ and $q=1$. It is obvious from Fig. 1, that the behavior of the black hole temperature significantly depends on $\alpha$ for small $S$. Accordingly, we expand the temperature of the charged dilaton AdS black hole for small entropy as follows:

(i) For $0<\alpha<1 / \sqrt{3} \approx 0.58$,

$$
\begin{aligned}
T= & \frac{\left(3 \alpha^{2}-1\right)\left(\alpha^{2}+1\right)^{1 /\left(\alpha^{2}+1\right)-1} q^{2 /\left(\alpha^{2}+1\right)}}{\pi 2^{4 /\left(\alpha^{2}+1\right)+1} l^{2-2 /\left(\alpha^{2}+1\right)} S^{2 /\left(\alpha^{2}+1\right)-1 / 2}} \\
& +\mathcal{O}\left(S^{2 /\left(\alpha^{2}+1\right)-5 / 2}\right)
\end{aligned}
$$

the black hole is "Reissner-Nordstrom-AdS" (RN) type in which with decreasing entropy, the temperature goes over zero. 


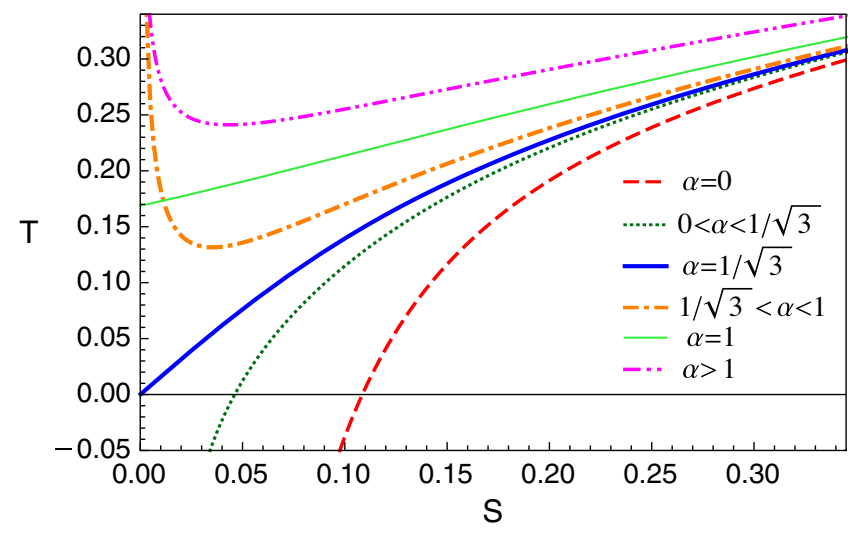

FIG. 1. T-S diagram of charged dilaton AdS black hole. This figure shows the remarkable influence of the coupling constant $\alpha$ on the temperature. Here, we have set $l=1$ and $q=1$.

(ii) For $\alpha=1 / \sqrt{3}$,

$$
\begin{aligned}
T= & \frac{3\left(3 l^{2}+12 q^{2}-l \sqrt{48 q^{2}+9 l^{2}}\right)}{2 \pi l^{4} q^{2}\left(\sqrt{9+48 q^{2} / l^{2}}-3\right)^{3 / 2}} \\
& \times \sqrt{3 l^{2}+8 q^{2}+l \sqrt{48 q^{2}+9 l^{2}}}+\mathcal{O}\left(S^{3}\right),
\end{aligned}
$$

where the dilaton black hole has zero temperature at the vanishing entropy limit. This $\alpha$ may be called the "marginal coupling constant" $\left(\alpha_{m}\right)$.

(iii) For $1 / \sqrt{3}<\alpha<1$,

$$
T=\frac{\left(3 \alpha^{2}-1\right)\left(\alpha^{2}+1\right)^{1 /\left(2 \alpha^{2}\right)-1}}{\pi 2^{1 / \alpha^{2}+1} l^{2} q^{-1 / \alpha^{2}} S^{1 /\left(2 \alpha^{2}\right)-1 / 2}}+\mathcal{O}\left(S^{1 /\left(2 \alpha^{2}\right)-1 / 2}\right),
$$

black hole is "Schwarzschild-AdS" (Schw)-type. In this case, black hole solution does not exist in the low-temperature regime.

(iv) For $\alpha=1$,

$$
T=\frac{l^{2}+2 q^{2}}{4 \sqrt{2} l^{2} \pi q}+\mathcal{O}(S)
$$

which is the "spatial" case where the dilaton black hole has finite temperature at $S=0$.

(v) For $\alpha>1$,

$$
T=\frac{2^{1 / \alpha^{2}-3}\left(\alpha^{2}+1\right)^{-1 /\left(2 \alpha^{2}\right)}}{\pi q^{1 /\left(\alpha^{2}\right)} S^{1 / 2-1 /\left(2 \alpha^{2}\right)}}+\mathcal{O}\left(S^{1 / 2-1 /\left(2 \alpha^{2}\right)}\right)
$$

black hole is Schw-type again. As can be seen from Fig. 1, the right branch of isocharge for Schw-type black hole is locally stable, i.e., the specific heat at constant charge is positive. On the other hand, the large entropy limit of the temperature is

$$
T \approx 3 \frac{\sqrt{S}}{2 \pi l^{2}} \quad \Rightarrow \quad C_{q}=2 S>0
$$

which is independent of the charge and dilaton coupling constant and always yields a thermal stable system.

The thermal stability of a charged AdS dilaton black hole was analyzed by means of $\left(\partial^{2} M / \partial S^{2}\right)_{q}$ for higher dimensional spacetimes at the fixed $l$ and $b$ in Ref. [23]. By fixing $l$ and $b$, the charge of black hole is determined as a function of $\rho_{+}$and $\alpha$ using the black hole horizon relation $f\left(\rho_{+}\right)=0$ and Eq. (7),

$$
q=q\left(\rho_{+}, \alpha\right)
$$

Indeed, charge $(q)$ is not fixed in the corresponding figures of Ref. [23]. Here, we classify the four dimensional charge AdS dilaton black holes by examining the behavior of the specific heat, Eq. (13), at fixed $l$ (i.e., cosmological constant) and $q$, for various values of $\alpha$. Such classification was not performed in previous studies [23,24].

In what follows, we are going to obtain the critical point, which corresponds to a second order phase transition, for various type of charged dilaton AdS black holes. For fixed $q$ and $l$, the value of the critical point is characterized by the inflection point

$$
\left.\frac{\partial T}{\partial S}\right|_{q_{c}}=0,\left.\quad \frac{\partial^{2} T}{\partial S^{2}}\right|_{q_{c}}=0 .
$$

To calculate the above expressions from Eq. (10) and (11), the following relation is used

$$
\left.\frac{\partial T}{\partial S}\right|_{q}=\frac{\left.\frac{\partial T}{\partial \rho_{+}}\right|_{b, q}+\left.\left.\frac{\partial T}{\partial b}\right|_{\rho_{+}, q} \frac{\partial b}{\partial \rho_{+}}\right|_{q}}{\left.\frac{\partial S}{\partial \rho_{+}}\right|_{b}+\left.\left.\frac{\partial S}{\partial b}\right|_{\rho_{+}} \frac{\partial b}{\partial \rho_{+}}\right|_{q}},
$$

where

$$
\left.\frac{\partial b}{\partial \rho_{+}}\right|_{q}=-\frac{\left.\frac{\partial f\left(\rho_{+}\right)}{\partial \rho_{+}}\right|_{b, q}}{\left.\frac{\partial f\left(\rho_{+}\right)}{\partial b}\right|_{\rho_{+}, q}} .
$$

Due to the complicated form of Eqs. (10) and (21), it is almost impossible to obtain the critical values, analytically. Hence, we numerically solve the set of Eq. (21) for a given value of $\alpha$. The calculated values of the critical quantities, such as $q_{c}, T_{c}, \rho_{+c}$, and $S_{c}$, for various $\alpha$ are illustrated in Figs. 2 and 3. 


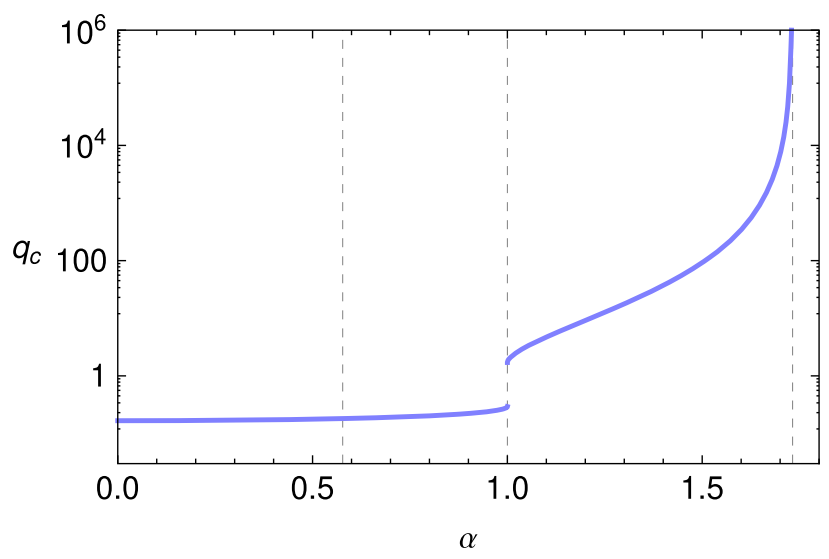

(a) $q_{c}$ versus $\alpha$

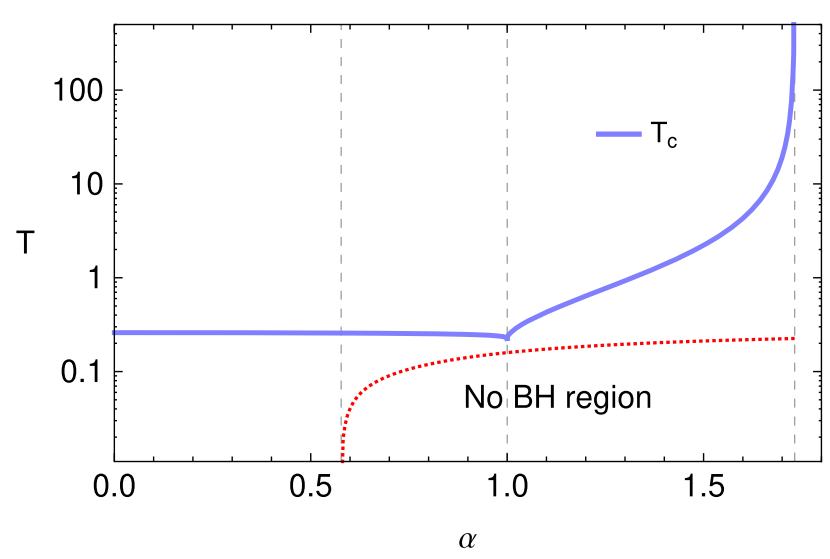

(b) $T_{c}$ versus $\alpha$

FIG. 2. The behaviors of the critical electric charge $\left(q_{c}\right)$ and critical temperature $\left(T_{c}\right)$ versus $\alpha$. The no BH region corresponds to no BH solution. The vertical dashed lines mark the values of $\alpha=1 / \sqrt{3}, \alpha=1$, and $\alpha=\sqrt{3}$. We use the logarithmic scales on the vertical axis and set $l=1$.

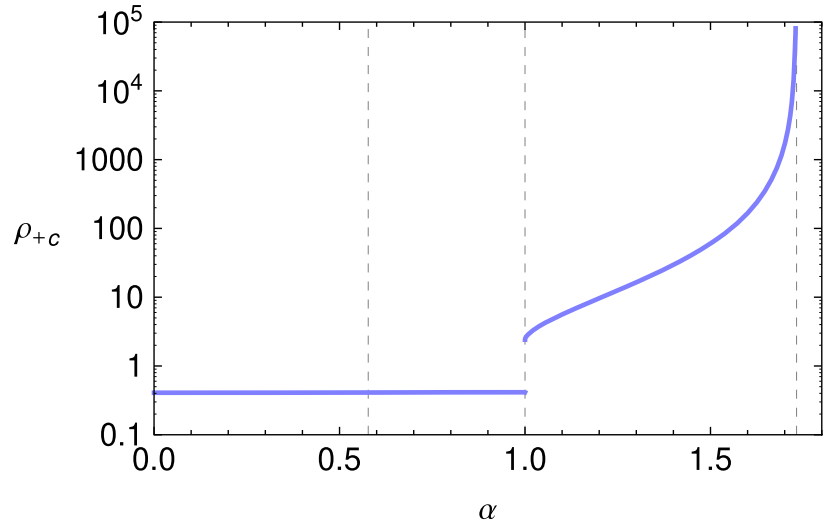

(a) $\rho_{+c}$ versus $\alpha$

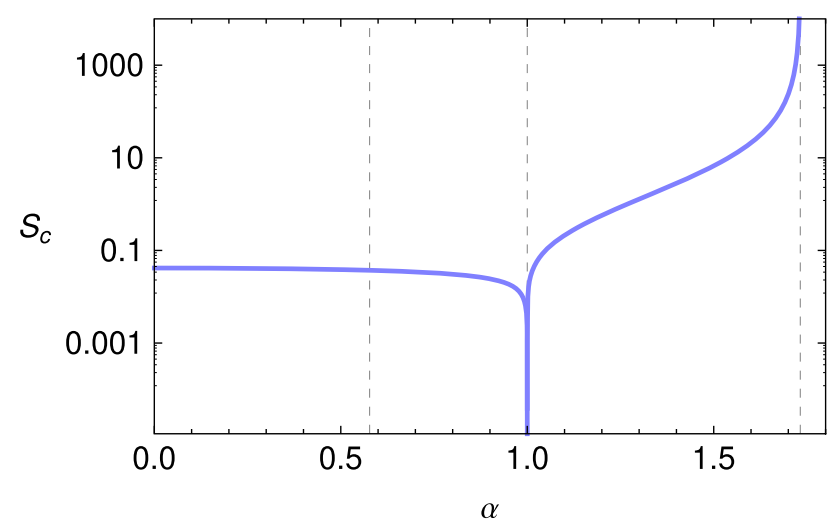

(b) $S_{c}$ versus $\alpha$

FIG. 3. The behaviors of the critical event horizon radius $\left(\rho_{+c}\right)$ and critical entropy $\left(S_{c}\right)$ versus $\alpha$. The vertical dashed lines mark the values of $\alpha=1 / \sqrt{3}, \alpha=1$ and $\alpha=\sqrt{3}$. We use the logarithmic scales on the vertical axis and set $l=1$.

We observe that there is no critical point for charged dilaton AdS black hole in cases where $\alpha=1$ and $\alpha \geq \sqrt{3} \approx 1.73$. As one can see from Figs. 2 and 3 , for $0<\alpha<1$, the dilaton coupling parameter $(\alpha)$ does not significantly affect the critical quantities, except entropy which abruptly decreases close to 1 . Figure 2(b) shows that the critical point occurs in the $\mathrm{RN}$-type of black hole when $0 \leq \alpha<1 / \sqrt{3}$, whereas for $1 / \sqrt{3}<\alpha<1$ it occurs in Schw-type where there is a lower bound on temperature of the black hole. As expected from Figs. 2 and 3, in the absence of dilaton field $(\alpha=0)$, the critical quantities reduce to those of charged AdS black hole [4]. In case of $1<\alpha<\sqrt{3}$, with increasing $\alpha$, the values of critical quantities increase and diverge for $\alpha \rightarrow \sqrt{3}$. It should also be pointed out that in the range $1<\alpha<\sqrt{3}$, the critical behavior happens in Schw-type region.
In order to fully obtain phase transition and examine phase structure of charged dilaton AdS black holes, we shall study the behavior of Helmholtz free energy in the next section.

\section{HELMHOLTZ FREE ENERGY}

The general thermodynamic description of charged dilaton AdS black hole is provided by studying the Helmholtz free energy which exhibits the global stable state. Indeed, the Helmholtz free energy is an appropriate thermodynamic potential to characterize equilibrium processes at constant temperature and charge in the canonical ensemble [28]. The Helmholtz free energy for a fixed AdS radius regime can be obtained through the Legendre transformation of the mass $M$. Thus, the Helmholtz free energy per unit volume $\omega$ is given 


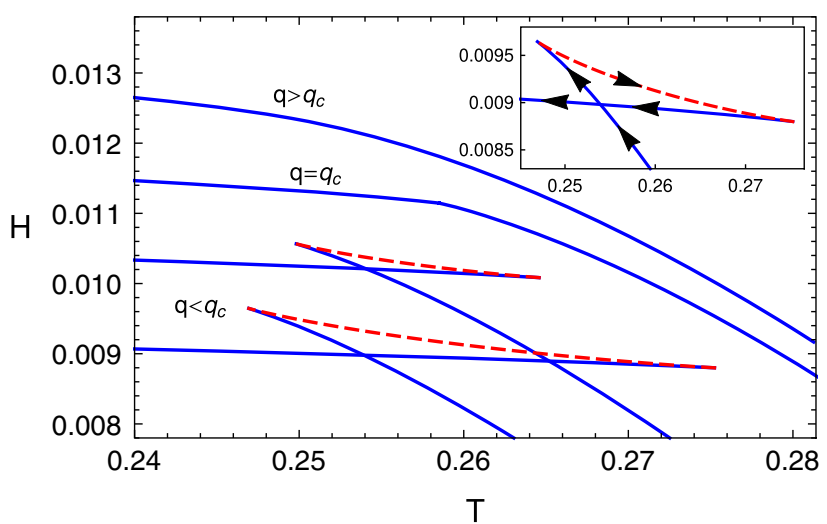

(a) $\alpha=0.5 \in[0,1 / \sqrt{ } 3]$

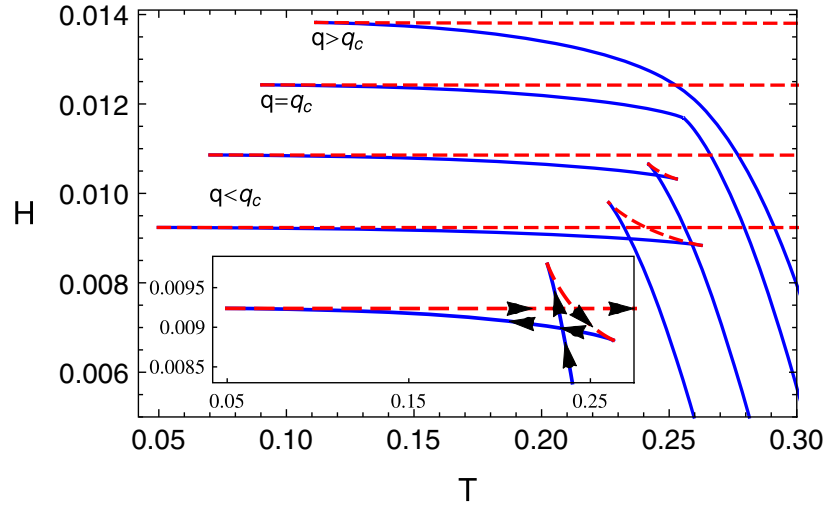

(b) $\alpha=0.7 \in(1 / \sqrt{ } 3,1)$

FIG. 4. Helmholtz free energy as a function of temperature for $l=1$ and various values of $q$. For $q<q_{c}$, the system undergoes a first order phase transition between SBH and LBH. The positive (negative) sign of $C_{q}$ is identified by the blue solid (dashed red) line. Insets: Black arrows show the direction of the decreasing $\rho_{+}$. The curves are shifted for clarity.

$$
\begin{aligned}
H(T, q) & =M-T S \\
& =\frac{l\left(\Upsilon\left[3-4 \Gamma-\alpha^{2}\right]+2\left(\alpha^{2}-1\right)+\alpha^{2}+5\right)}{32 \sqrt{2} \pi\left(\alpha^{2}+1\right) \Gamma^{3 / 2-2 /\left(\alpha^{2}+1\right)}(\Upsilon-1)^{-1 / 2}},
\end{aligned}
$$

where

$$
\Upsilon \equiv \sqrt{1+\frac{4 q^{2}\left(\alpha^{2}+1\right) \Gamma^{3-4 /\left(\alpha^{2}+1\right)}}{l^{2}(1-\Gamma)}},
$$

and $\Gamma=1-b / \rho_{+}$, thus we have $\Gamma=\Gamma(T, q)$. Notice that the reality condition of the black hole solutions $(\rho>b)$ leads to the constraint $0<\Gamma<1$.

The behavior of Helmholtz free energy in terms of the temperature $T$ for $\alpha=0.5,0.7$, and 1.3 are depicted in Figs. 4 and 5, for different values of charge $q$. From these

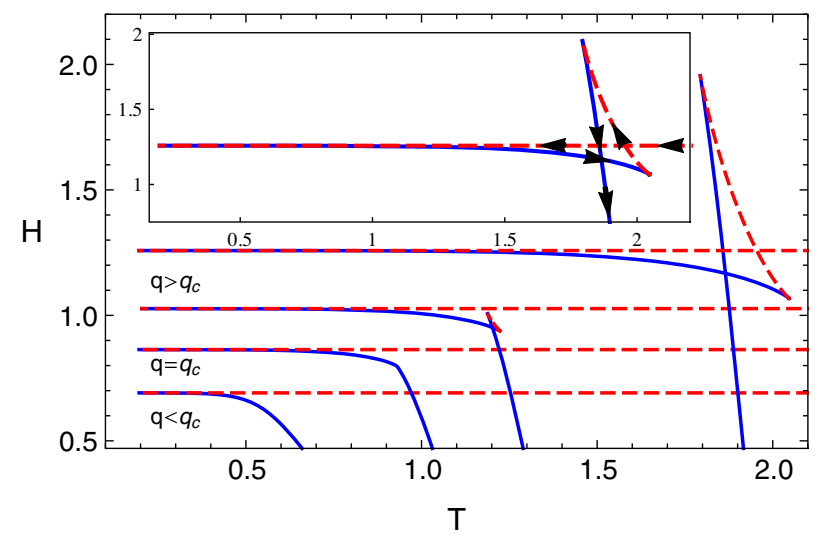

FIG. 5. Helmholtz free energy as a function of temperature for $l=1, \alpha=1.3 \in(1, \sqrt{3})$ and various values of $q$. For $q>q_{c}$, the system undergoes a first order phase transition between SBH and $\mathrm{LBH}$. The positive (negative) sign of $C_{q}$ is identified by the blue solid (dashed red) line. Inset: Black arrows show the direction of the decreasing $\rho_{+}$. The curves are shifted for clarity. figures, it can be seen that the charge dependence of Helmholtz free energy is strongly affected by the dilaton coupling parameter $\alpha$. In case of $\alpha=0.5 \in[0,1 / \sqrt{3}]$, where charged dilaton AdS black hole is RN-type, the Helmholtz free energy is single value in temperature for $q>q_{c}$ [see Fig. 4(a)]. In this case, back hole is locally stable $\left(C_{q}>0\right)$ which is indicated by the solid blue line in Fig. 4(a). On the other hand, when $q<q_{c}$, black hole becomes thermodynamically unstable where the Helmholtz free energy is multi-valued in the certain range of temperature. This corresponds to $C_{q}<0$ which is shown by dashed-red line in Fig. 4(a). A close up of such a curve is illustrated in the inset of Fig. 4(a), where the arrows indicate the direction of the decreasing black hole event horizon $\left(\rho_{+}\right)$. Decreasing the temperature of the system follows the lower solid blue curve, which corresponds to the lowest Helmholtz free energy, until it crosses the upper solid blue curve. In this position, system enters to the left solid blue curve with a first order large black hole $(\mathrm{LBH}) / \mathrm{small}$ black hole $(\mathrm{SBH})$ phase transition which is accompanied by a discontinuity in the slop of Helmholtz free energy. ${ }^{1}$ The small (large) is referred to the size of horizon radius $\rho_{+}$. For $\alpha=0.7 \in(1 / \sqrt{3}, 1)$ case, as illustrated in Fig. 4(b), charged dilaton AdS black hole is Schw-type where the lower (upper) branch of the Helmholtz free energy is thermodynamically stable (unstable) with $C_{q}>0\left(C_{q}<0\right)$ for $q>q_{c}$. At $q=q_{c}$, there is a critical point in the lower branch of $H$. For $q<q_{c}$, Helmholtz energy becomes multivalued with negative $C_{q}$ in the lower branch. For such a case, the direction of decreasing $\rho_{+}$is marked with arrows in the inset of Fig. 4(b). As temperature of black hole decreases, the horizon $\left(\rho_{+}\right)$

\footnotetext{
${ }^{1} \mathrm{~A}$ first order phase transition occurs when there exist a discontinuity in the first derivative of Helmholtz free energy with respect to temperature which is entropy, i.e., $(\partial H / \partial T)=S$.
} 


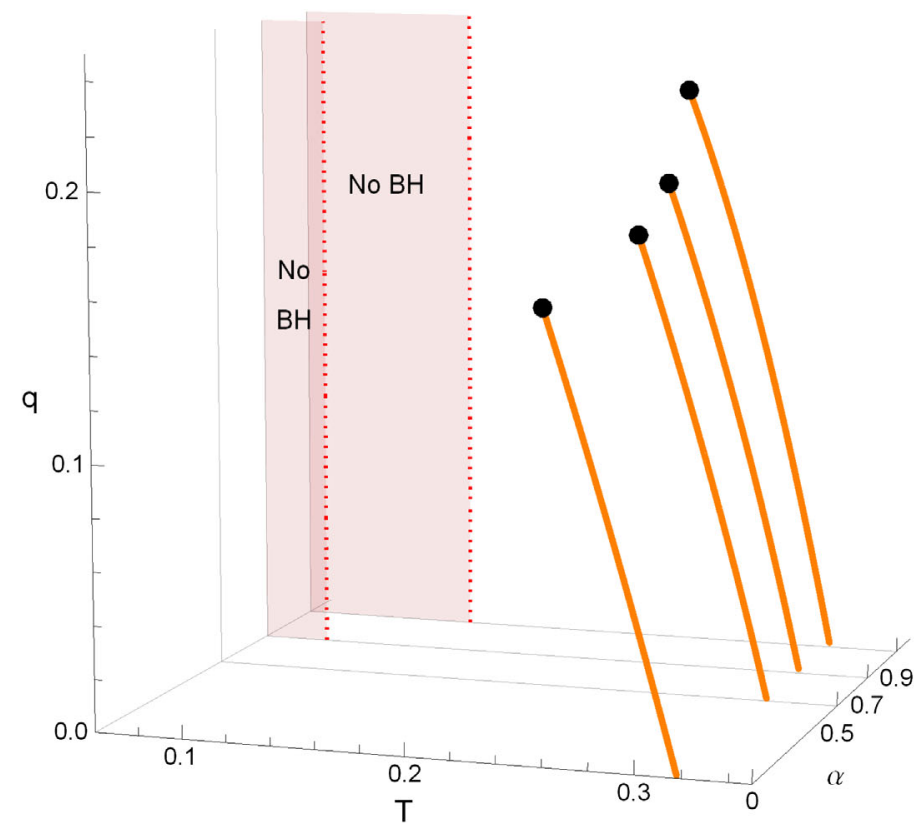

(a)

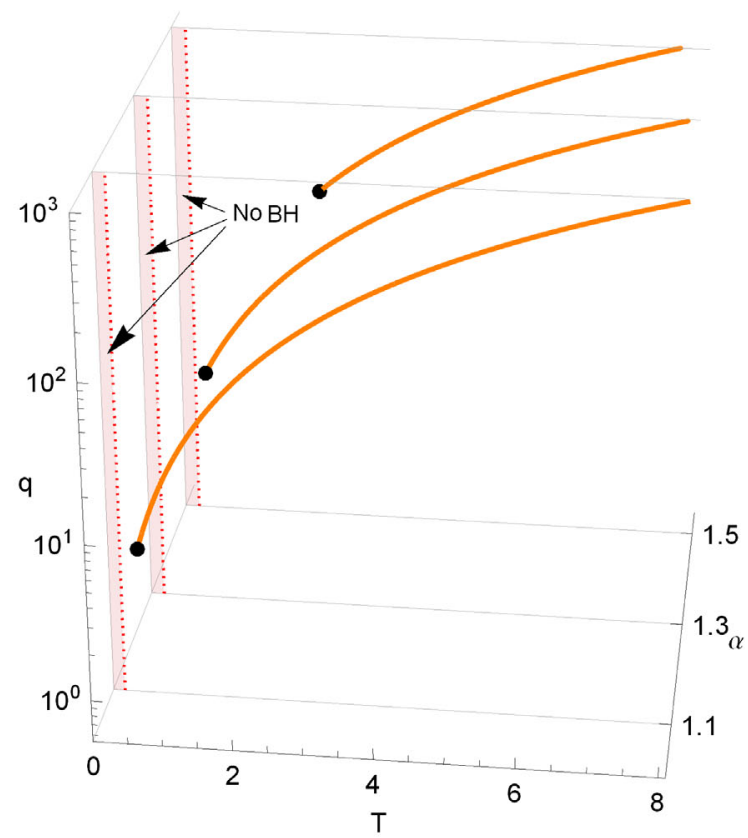

(b)

FIG. 6. SBH/LBH phase diagram for $l=1$ and various values of $\alpha$. The critical points and first order phase transition curves are highlighted by the black solid circle and solid orange line, respectively. At low temperature, no $\mathrm{BH}$ regions correspond to no black hole solution. We use the logarithmic scale on $q$ axis in Fig. (b).

decreases along the lowest stable (solid blue) branch of the curve until two stable branches cross each other. At this point, black hole enters to the solid blue branch with the discontinuous change in the slope of Helmholtz free energy, indicating a first order phase transition which occurs between the LBH and SBH. Further decreasing $T$, black hole follows the left solid blue branch until the end. For $\alpha=1.3 \in(1, \sqrt{3})$, a novel behavior happens in for Schw-type black hole (see Fig. 5). Indeed, in contrast to what occurs in Fig. 4(b), in this case a first order phase transition takes place between SBH and LBH for $q>q_{c}$. In the inset of Fig. 5, the decrease in $\rho_{+}$is denoted by the arrows, which is opposite to the previous case, i.e., the inset in Fig. 4(b). This behavior has not been observed in previous studies on phase transition of charged AdS black holes $[26,33]$. It is notable to mention that we do not find any other phase transition for charged dilaton AdS black hole.

The corresponding SBH/LBH phase diagram of dilaton AdS black hole for different values of the dilaton parameter $\alpha$ is sketched in Fig. 6. It is clear from Fig. 6 that the critical points are denoted by black spots at the end of the first order phase transition curves (orange). In Fig. 6(a), the first order phase transition curves separate the SBH from LBH for $q<q_{c}$, while in Fig. 6(b), these curves distinguish the SBH from LBH for $q>q_{c}$. Also, no $\mathrm{BH}$ regions implies that no $\mathrm{BH}$ solutions exist at the low temperature.

\section{SUMMARY}

To sum up, we have revisited critical behavior and phase structure of charged dilaton black holes in the background of AdS spaces. The motivation for study phase behavior of dilaton black holes in AdS spacetime is mainly inspired by AdS/CFT correspondence and is expected to shed light on the microscopic structure of black holes. We adopted the view point that cosmological constant can be regarded as a fixed parameter, while the charge of the black hole varies.

To understand the impact of the dilaton field on the heat capacity, $C_{q}$, which determines the local thermodynamic stability of the system, we have studied the behavior of the temperature $T$ as a function of entropy $S$ for different values of $\alpha$. By expanding $T$ for small values of $S$, we have distinguished several black hole systems, with thermal stability/instability, depending on the values of $\alpha$. In order to obtain the coordinates of the critical point, we numerically solved the system of equations and plotted the quantities at the critical point in terms of $\alpha$. We observed that there is no critical point in cases with $\alpha=1$ and $\alpha \geq \sqrt{3}$, while for $0<\alpha<1$, the critical quantities are not significantly affected by $\alpha$, except entropy which abruptly decreases close to 1 . In the absence of dilaton field $(\alpha=0)$, the critical quantities reduce to those of charged AdS black hole.

We have also studied the Gibss free energy, which exhibits the global stable state of the system, for different values of $\alpha$ and $q$. We have realized several cases, depending on $\alpha$, including whether or not the Helmholtz free 
energy is single/multi-valued and whether or not the system is thermally stable/unstable. Interestingly enough, we observed that unstable behavior of the Helmholtz free energy for $q<q_{c}$ exhibits a first order (discontinuous) phase transition between SBH and LBH for $0 \leq \alpha<1$. For $1<\alpha<\sqrt{3}$, however, a novel first order phase transition happens between SBH and LBH provided $q>q_{c}$. The later has not been observed in the previous studies on phase transition of charged AdS black holes and is one of the new result of the present work.

\section{ACKNOWLEDGMENTS}

We are grateful to the Research Council of Shiraz University. This work has been supported financially by Research Institute for Astronomy and Astrophysics of Maragha, Iran. A. S. thanks Hermann Nicolai and MaxPlanck Institute for Gravitational Physics (AEI), for hospitality.

\section{APPENDIX: MASS OF DILATON BLACK HOLE IN AdS SPACE}

To obtain the mass of a charged dilaton AdS black hole in four dimensions, we use the background subtraction method of the Brown-York which is based on quasilocal concept $[23,38]$. Within this formalism, the line element should be written in the form

$$
d s^{2}=-\mathcal{W}(\mathcal{R}) d t^{2}+\frac{d \mathcal{R}^{2}}{\mathcal{V}(\mathcal{R})}+\mathcal{R}^{2} d \Omega^{2} .
$$

In order to obtain the metric Eq. (A1) from Eq. (3), we perform the following transformation

$$
\mathcal{R}=\rho\left(1-\frac{b}{\rho}\right)^{\gamma / 2}
$$

such that, the metric functions $\mathcal{W}$ and $\mathcal{V}$ are written as

$$
\begin{aligned}
\mathcal{W}(\mathcal{R}) & =f(\rho(\mathcal{R})), \\
\mathcal{V}(R) & =f(\rho(\mathcal{R}))\left(\frac{d \mathcal{R}}{d \rho}\right)^{2} \\
& =\left[1+\frac{b(\gamma-2)}{2 \rho}\right]^{2}\left(1-\frac{b}{\rho}\right)^{\gamma-2} f(\rho(\mathcal{R})),
\end{aligned}
$$

where $f$ is given by Eq. (4). According to the metric Eq. (4), the background metric is chosen to be of the form

$$
\begin{aligned}
\mathcal{W}_{0}(\mathcal{R}) & =\mathcal{V}_{0}(\mathcal{R})=f_{0}(\rho(\mathcal{R})) \\
& =1+\frac{\rho^{2}}{l^{2}}-\frac{2 \alpha^{2} b \rho}{l^{2}\left(\alpha^{2}+1\right)}+\frac{\alpha^{4} b^{2}}{l^{2}\left(\alpha^{2}+1\right)^{2}}
\end{aligned}
$$

To compute the mass of the space time, one should choose a timelike Killing vector $\xi$ on the boundary surface $\mathcal{B}$ of the space time Eq. (A1). Then, the quasilocal mass can be calculated via

$M=\frac{1}{8 \pi} \int_{\mathcal{B}} d^{2} \varphi \sqrt{\sigma}\left\{\left(K_{a b}-K h_{a b}\right)-\left(K_{a b}^{0}-K^{0} h_{a b}^{0}\right)\right\} n^{a} \xi^{b}$,

where $\sigma$ is the determinant of the metric $\sigma_{a b}$ of the boundary $\mathcal{B}, n^{a}$ is the timelike unit normal vector to the boundary and $K_{a b}^{0}$ is the extrinsic curvature of the background metric. In the context of the counterterm method, the limit in which the boundary becomes infinity, $\mathcal{B}_{\infty}$, is taken, and the counterterm prescription ensures that the action and mass are finite. By using the above modified Brown-York formalism, one can obtain the mass of the AdS dilaton black hole per unit volume as

$$
M=\frac{1}{8 \pi}\left(c-b \frac{\alpha^{2}-1}{\alpha^{2}+1}\right) .
$$

In the absence of the dilaton $(\alpha=0)$, this expression reduces to the mass of the AdS black hole in four dimensions.
[1] S. Hawking and D. N. Page, Thermodynamics of black holes in anti-de Sitter space, Commun. Math. Phys. 87, 577 (1983).

[2] E. Witten, Anti-de Sitter space, thermal phase transition, and confinement in gauge theories, Adv. Theor. Math. Phys. 2, 505 (1998).

[3] B. P. Dolan, The cosmological constant and the black hole equation of state, Classical Quantum Gravity 28, 125020 (2011); Pressure and volume in the first law of black hole thermodynamics, Classical Quantum Gravity 28, 235017 (2011).
[4] D. Kubiznak and R. B. Mann, P-V criticality of charged AdS black holes, J. High Energy Phys. 07 (2012) 033.

[5] D. Kastor, S. Ray, and J. Traschen, Enthalpy and the mechanics of AdS black holes, Classical Quantum Gravity 26, 195011 (2009).

[6] S. H. Hendi and M. H. Vahidinia, Extended phase space thermodynamics and P-V criticality of black holes with a nonlinear source, Phys. Rev. D 88, 084045 (2013).

[7] M. B. Jahani Poshteh, B. Mirza, and Z. Sherkatghanad, Phase transition, critical behavior, and critical exponents of Myers-Perry black holes, Phys. Rev. D 88, 024005 (2013). 
[8] Z. Sherkatghanad, B. Mirza, Z. Mirzaeyan, and S. A. H. Mansoori, Critical behaviors and phase transitions of black holes in higher order gravities and extended phase spaces, Int. J. Mod. Phys. D 26, 1750017 (2017).

[9] R. Banerjee and D. Roychowdhury, Critical phenomena in Born-Infeld AdS black holes, Phys. Rev. D 85, 044040 (2012); Critical behavior of Born-Infeld AdS black holes in higher dimensions, Phys. Rev. D 85, 104043 (2012).

[10] M. H. Dehghani, S. Kamrani, and A. Sheykhi, P-V criticality of charged dilatonic black holes, Phys. Rev. D 90, 104020 (2014).

[11] M. H. Dehghani, A. Sheykhi, and Z. Dayyani, Critical behavior of Born-Infeld dilaton black holes, Phys. Rev. D 93, 024022 (2016).

[12] Z. Dayyani, A. Sheykhi, and M. H. Dehghani, Counterterm method in dilaton gravity and the critical behavior of dilaton black holes with power-Maxwell field, Phys. Rev. D 95, 084004 (2017).

[13] Z. Dayyani, A. Sheykhi, M. H. Dehghani, and S. Hajkhalili, Critical behavior and phase transition of dilaton black holes with nonlinear electrodynamics, Eur. Phys. J. C 78, 152 (2018).

[14] B. R. Majhi and S. Samanta, P-V criticality of AdS black holes in a general framework, Phys. Lett. B 773, 203 (2017).

[15] R. A. Hennigar, R. B. Mann, and E. Tjoa, Superfluid Black Holes, Phys. Rev. Lett. 118, 021301 (2017).

[16] A. Dehghani and S. H. Hendi, Charged black hole chemistry with massive gravitons, Classical Quantum Gravity 37, 024001 (2020).

[17] S. Gunasekaran, R. B. Mann, and D. Kubiznak, Extended phase space thermodynamics for charged and rotating black holes and Born-Infeld vacuum polarization, J. High Energy Phys. 11 (2012) 110.

[18] N. Altamirano, D. Kubiznak, and R. B. Mann, Reentrant phase transitions in rotating anti-de Sitter black holes, Phys. Rev. D 88, 101502 (2013).

[19] S. H. Hendi and M. Momennia, Reentrant phase transition of Born-Infeld-dilaton black holes, Eur. Phys. J. C 78, 800 (2018).

[20] M. K. Zangeneh, A. Dehyadegari, A. Sheykhi, and R. B. Mann, Microscopic origin of black hole reentrant phase transitions, Phys. Rev. D 97, 084054 (2018).

[21] S. W. Wei and Y.X. Liu, Insight into the Microscopic Structure of an AdS Black Hole from Thermodynamical Phase Transition, Phys. Rev. Lett. 115, 111302 (2015); Erratum, Phys. Rev. Lett. 116, 169903 (2016).

[22] M. K. Zangeneh, A. Dehyadegari, and A. Sheykhi, Comment on "Insight into the microscopic structure of an AdS black hole from a thermodynamical phase transition", arXiv:1602.03711.
[23] A. Sheykhi, M. H. Dehghani, and S. H. Hendi, Thermodynamic instability of charged dilaton black holes in AdS spaces, Phys. Rev. D 81, 084040 (2010).

[24] A.-C. Li, H.-Q. Shi, and D.-F. Zeng, Phase structure and QNMs of a charged AdS dilaton black hole, Phys. Rev. D 97, 026014 (2018).

[25] A. Dehyadegari, A. Sheykhi, and A. Montakhab, Novel phase transition in charged dilaton black holes, Phys. Rev. D 96, 084012 (2017).

[26] A. Dehyadegari, A. Sheykhi, and A. Montakhab, Critical behaviour and microscopic structure of charged AdS black holes via an alternative phase space, Phys. Lett. B 768, 235 (2017).

[27] A. Chamblin, R. Emparan, C. V. Johnson, and R. C. Myers, Charged AdS black holes and catastrophic holography, Phys. Rev. D 60, 064018 (1999).

[28] A. Chamblin, R. Emparan, C. V. Johnson, and R. C. Myers, Holography, thermodynamics and fluctuations of charged AdS black holes, Phys. Rev. D 60, 104026 (1999).

[29] Z. Dayyani and A. Sheykhi, Critical behavior of Lifshitz dilaton black holes, Phys. Rev. D 98, 104026 (2018).

[30] H. Yazdikarimi, A. Sheykhi, and Z. Dayyani, Critical behavior of Gauss-Bonnet black holes via an alternative phase space, Phys. Rev. D 99, 124017 (2019).

[31] A. Sheykhi, M. Arab, Z. Dayyani, and A. Dehyadegari, Alternative approach to the critical behavior and microscopic structure of the power-Maxwell black holes, Phys. Rev. D 101, 064019 (2020).

[32] A. Dehyadegari, B. R. Majhi, A. Sheykhi, and A. Montakhab, Universality class of alternative phase space and van der Waals criticality, Phys. Lett. B 791, 30 (2019).

[33] A. Dehyadegari and A. Sheykhi, Reentrant phase transition of Born-Infeld-AdS black holes, Phys. Rev. D 98, 024011 (2018).

[34] M. K. Zangeneh, A. Dehyadegari, M. R. Mehdizadeh, B. Wang, and A. Sheykhi, Thermodynamics, phase transitions and Ruppeiner geometry for Einstein-dilaton Lifshitz black holes in the presence of Maxwell and Born-Infeld electrodynamics, Eur. Phys. J. C 77, 423 (2017).

[35] C. J. Gao and S. N. Zhang, Dilaton black holes in de Sitter or anti-de Sitter Universe, Phys. Rev. D 70, 124019 (2004).

[36] C. J. Gao and S. N. Zhang, Higher dimensional dilaton black holes with cosmological constant, Phys. Lett. B 605 , 185 (2005).

[37] C. J. Gao and S. N. Zhang, Topological black holes in dilaton gravity theory, Phys. Lett. B 612, 127 (2005).

[38] S. H. Hendi, A. Sheykhi, and M. H. Dehghani, Thermodynamics of higher dimensional topological charged AdS black branes in dilaton gravity, Eur. Phys. J. C 70, 703 (2010).

[39] A. Sheykhi, Charged rotating dilaton black strings in AdS spaces, Phys. Rev. D 78, 064055 (2008). 